
cultures

Les cahiers de l'Acedle

\title{
Les enseignants face à la diversité linguistique
}

Vers une conception alterlinguistique de l'enseignement?

Florence Rémy-Thomas

\section{OpenEdition}

1 Journals

Édition électronique

URL : http://journals.openedition.org/rdlc/2927

DOI : $10.4000 /$ rdlc.2927

ISSN : 1958-5772

Éditeur

ACEDLE

Référence électronique

Florence Rémy-Thomas, "Les enseignants face à la diversité linguistique », Recherches en didactique des langues et des cultures [En ligne], 3 | 2008, mis en ligne le 30 juillet 2008, consulté le 19 avril 2019. URL : http://journals.openedition.org/rdlc/2927 ; DOI : 10.4000/rdlc.2927

Ce document a été généré automatiquement le 19 avril 2019.

\section{(c) (i) (9)}

Recherches en didactique des langues et des cultures is licensed under a Creative Commons AttributionNonCommercial-NoDerivatives 4.0 International License 


\section{Les enseignants face à la diversité linguistique}

Vers une conception alterlinguistique de l'enseignement?

Florence Rémy-Thomas

\section{Introduction}

1 L'approche de l'enseignement dont il va être question tout au long de cet article s'appuie sur une étude réalisée dans le cadre de deux modules de formation en didactique des langues et des cultures à l'université de Paris III - Sorbonne Nouvelle, assurés auprès d'enseignants ou futurs enseignants de français langue seconde et/ou étrangère qui se destinent à travailler avec un public dont les appartenances langagières sont multiples. L'objet de cette étude est de montrer comment faire évoluer des conceptions de l'enseignement/apprentissage des langues qui semblent au départ bien stabilisées. Pour ce faire, nous tenterons de tracer les contours d'une conception alterlinguistique de l'enseignement, à partir des réponses obtenues en début et en fin de parcours d'une part, par un groupe inscrit dans la mention FLE des licences ${ }^{1}$ ayant suivi un module d'anthropologie et communication, et d'autre part, par un groupe inscrit dans le diplôme universitaire de didactique des langues (DUDL) ${ }^{2}$ ayant été initié à une approche de la diversité linguistique et culturelle. L'objectif de cette conception à visée professionnelle est de faciliter la capacité à intégrer dans sa pratique enseignante une ouverture vis-à-vis de la langue et de la culture que l'on ne connaît pas ou peu, celle de l'individu dont la langue familiale n'est pas la langue de l'école, par la mise en place d'une approche plurielle des langues, telle qu'elle est décrite dans le projet d'Éveil aux langues (programme Evlang, Candelier 2003). En proposant de travailler à la prise en compte des langues exogènes présentes dans les classes accueillant un public qui possède d'autres langues que le français, nous tentons de comprendre comment des représentations parfois stéréotypées de l'altérité linguistique vont se déplacer vers des représentations plus dynamiques et plus flexibles adaptées aux besoins des élèves allophones. 
2 Une première analyse des représentations des enseignants vis-à-vis du rôle de "l'autre » langue dans l'apprentissage du français apparaît alors comme un point de départ essentiel pour cerner le rôle qu'ils attribuent à la langue première (désormais L1) dans le processus d'apprentissage des langues. Les notions d'altérité et d'interculturalité seront fortement sollicitées dans le travail d'analyse des résultats et nous conduira à une réflexion sur les implications didactiques que cela suppose de mettre en œuvre.

\section{Contexte d'apprentissage et altérité dans l'appropriation des langues}

3 Dans cette étude, il s'agit de comprendre ce qui influence de manière indirecte ou implicite les représentations des enseignants quant aux buts qu'ils poursuivent lorsqu'ils enseignent le français en contexte linguistiquement hétérogène. Notre analyse se focalisera sur la question de l'altérité linguistique et de l'influence du macro-contexte d'apprentissage sur la culture didactique à laquelle les enseignants en formation de notre échantillon se réfèrent. Selon qu'ils préconisent une approche cloisonnée ou une approche inclusive de l'enseignement des langues, nous postulons que la culture éducative $^{3}$ à laquelle appartiennent les enseignants est fortement empreinte de l'idéologie monolingue ${ }^{4}$ qui caractérise le système éducatif français et ne peut être remise en cause que par une formation impliquant une approche de la diversité linguistique et culturelle, dépassant ainsi les objectifs de la pédagogie interculturelle ${ }^{5}$.

4 Le cadre théorique auquel nous nous référons est celui que Porquier \& Py (2004) mobilisent pour définir le contexte d'apprentissage qui influence toute situation d'appropriation d'une langue - le contexte macro et le contexte micro ${ }^{6}$-. Sans exclure le contexte micro de la situation d'enseignement/apprentissage, nous porterons plus particulièrement notre attention sur la dimension macro pour expliquer que la formation antérieure des enseignants fait référence aux facteurs sociaux qui sont déterminés par la politique éducative mise en œuvre dans le système éducatif français. Nous pensons, en effet, que les rôles attribués d'une part, à la langue de l'école dans la société et ceux assignés, d'autre part, à l'apprentissage des langues à l'école sont déterminants dans la façon d'appréhender la diversité linguistique et culturelle. Nous nous inscrivons par ailleurs dans le sillage des travaux grenoblois menés depuis plus de vingt-années dans le laboratoire Linguistique et Didactique des Langues Étrangères et Maternelles (Lidilem), neuchâtelois (Matthey, 1997b) et français (Candelier, 1997) qui ont fait l'objet d'un ouvrage de synthèse (Moore, 2003), portant sur la relation entre représentations et apprentissage des langues.

\section{Protocole de l'étude : la meilleure façon d'apprendre une langue}

Cette étude est fondée sur un protocole commun aux deux groupes de notre échantillon, développé dans le but de recueillir les représentations des enseignants ou futurs enseignants en début de formation et en fin de parcours sous la forme d'un bilan. Le questionnaire distribué ${ }^{7}$ porte sur les croyances épistémologiques (croyances sur l'apprentissage des langues en général) destiné à identifier une " matrice disciplinaire ${ }^{8}$ sur laquelle les enseignants ou futurs enseignants s'appuient pour définir leur conception de l'apprentissage des langues. Nous avons donné aux étudiants l'occasion d'expliquer comment ils avaient interprété ces questions dans le cadre d'une analyse qui a fait l'objet 
d'une correction collective, destinée à montrer qu'il ne s'agissait en aucun cas d'une évaluation mais d'un exercice pour les sensibiliser aux questions qui allaient être abordées en cours.

6 Les enseignants des deux formations ont donc répondu à ce questionnaire dont nous n'avons retenu dans un premier temps que les réponses aux questions portant sur la meilleure façon d'apprendre une langue et sur les finalités de l'apprentissage des langues. Un point de vue - celui de l'enseignant ou futur enseignant - est plus particulièrement étudié, selon une démarche qualitative, dans le but d'identifier ce que nous appelons l'idéologie éducative de l'enseignant, qui affiche selon notre analyse, soit une appartenance à la tradition monolingue de l'apprentissage qui cloisonne les langues, soit une adhésion à la valorisation du plurilinguisme comme proposition pour enrichir son répertoire d'enseignant. Nous avons choisi pour vérifier ces hypothèses de poser des questions ouvertes concernant l'apprentissage des langues à dix enseignants ou futurs enseignants du premier et du second degré que nous avons sélectionnés en fonction de leur cursus (mention FLE pour le second degré et DUDL pour le premier degré) et de leur plus ou moins longue expérience de l'enseignement des langues, afin de vérifier de quelle manière leurs propositions pouvaient mettre en évidence le rôle de la L1 dans le processus d'apprentissage.

\begin{tabular}{|l|l|l|l|l|}
\hline Formation & $\begin{array}{l}\text { Enseignants } \\
\text { novices }\end{array}$ & $\begin{array}{l}\text { Expérience de } \\
\text { l'enseignement du français } \\
\text { et/ou de l'enseignement des } \\
\text { langues }\end{array}$ & $\begin{array}{l}\text { Conception } \\
\text { inclusive }\end{array}$ & $\begin{array}{l}\text { Conception } \\
\text { cloisonnée }\end{array}$ \\
\hline $\begin{array}{l}\text { Mention FLE } \\
\text { Enseignants du } \\
\text { second degré }\end{array}$ & 3 & 7 & 0 & 10 \\
\hline $\begin{array}{l}\text { DUDL } \\
\text { Enseignants du } \\
\text { premier degré }\end{array}$ & 2 & 8 & 0 & 10 \\
\hline
\end{tabular}

Tableau - Profil des enseignants dans les deux formations.

\section{Entre rituels d'apprentissage et représentation idéalisée}

7 Le choix de retenir dans notre corpus la première réponse apportée par ces enseignants ou futurs enseignants à la question posée se justifie par l'idée qu'elle traduit une croyance et une référence forte à la conception de l'enseignement / apprentissage des langues. Il s'agira dans un second temps de voir dans quelle mesure la référence à une représentation idéalisée, celui du modèle du locuteur natif qui doit devenir un spécialiste de la langue enseignée, est un modèle prégnant parmi les enseignants ou futurs enseignants interrogés.

8 A la question portant sur la meilleure façon d'apprendre une langue, l'échantillon de la mention FLE préconise en début de formation dans sa grande majorité le bain linguistique ou l'immersion comme situation idéale de l'apprentissage d'une langue dont certains 
excluent totalement la L1 du processus. Pour ce qui concerne les enseignants ou futurs enseignants du premier degré, nous observons que la situation d'immersion est également majoritairement citée mais s'accompagne plus souvent d'une dimension ludique et rassurante, ce qui semble à priori en contradiction avec la situation d'immersion mais indique clairement la nécessité de s'adapter au public d'enfants.

Les exemples qui vont suivre tendent à confirmer que les enseignants ou futurs enseignants n'éprouvent pas le besoin d'avoir recours à la L1 car leur savoir académique

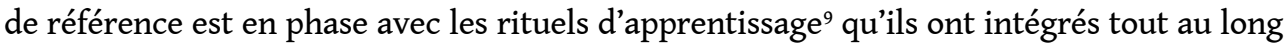
de leur scolarité. Si l'on considère que ces rituels ont été largement établis en référence à leur apprentissage du français et d'une langue étrangère dans le système éducatif français, il nous parait évident que le modèle construit est un modèle issu d'une approche cloisonnée, du fait qu'il n'existe pas dans leur parcours de modèle intégrant une réflexion commune entre ces apprentissages.

\section{Exemple 1 : Corpus Mention FLE}

1a. Ens. $7:$ : Il faut être avec des personnes qui parlent cette langue (i.e la langue cible) et qui ne parlent pas la mienne pour me forcer à parler.» Expérience de l'enseignement du français au collège/lycée (10 ans).

1b. Ens. 8 : «L'immersion dans le pays est selon moi la meilleure façon de connaître une langue. »Expérience de l'enseignement du français au collège/ lycée (8ans).

1c. Ens. 9: «Partir pour une durée de 6 mois ou 1 an minimum à l'étranger afin de maîtriser la langue en question en immersion totale, c'est-à-dire en communiquant avec les gens de ce pays du matin au soir, en évitant au maximum de parler sa langue maternelle avec des compatriotes rencontrés dans le pays. » Pas d'expérience de l'enseignement du français et/ou LVE au collège/lycée.

\section{Exemple 2 : Corpus DUDL}

2a. Ens. 1 : « de façon ludique, en immersion, à l'aide de supports divers, par la répétition.» Expérience de l'enseignement des langues aux enfants en institution scolaire (4 ans).

2b. Ens. 5 : « être immergé, en avoir l'utilité et en éprouver du plaisir. Si ce n'est pas le cas, lors de situation d'échec, être accompagné pour surmonter ses difficultés. » Pas d'expérience de l'enseignement des langues aux enfants.

10 Ces exemples illustrent le fait que les enseignants semblent se concentrer beaucoup plus sur l'aspect communication que l'immersion préfigure dans l'apprentissage sans prise en compte des bénéfices cognitifs que pourraient apporter la $\mathrm{L} 1^{10}$. Toutefois, dans les exemples contrastés, ci-dessous, l'immersion bien que considérée comme la situation répondant au mieux aux objectifs d'un apprentissage idéalisé - celui du bain linguistique apparait dans les exemples $3 a$. et $3 b$. comme ne pouvant être réalisé dans le cadre d'un apprentissage scolaire, alors que l'exemple 3c. renvoie à une conception scolaire de l'apprentissage comme l'usage du terme « grammaire » le laisse supposer.

\section{Exemple 3 :}

Corpus Mention FLE

3a. Ens. 1 : «Communiquer avec ses locuteurs [de la L2] et séjourner dans un lieu où elle est pratiquée rendent l'apprentissage plus vivant et naturel. » Pas d'expérience de l'enseignement du français et/ou d'une LVE au collège/ lycée.

Corpus DUDL 
3b. Ens. 10: "par une activité extra-scolaire (jeu, théâtre, chant, sport, dessin).» Expérience de l'enseignement des langues aux enfants en institution scolaire ( 6 ans, puis reconversion professionnelle).

3c. Ens. 3: " être immergé pour parler, avoir un bon livre de grammaire pour écrire. » Expérience de l'enseignement des langues aux enfants en institution scolaire (5ans).

\section{Une culture éducative monolingue vs une culture didactique cloisonnée}

11 La référence à la notion de culture éducative nous permet de poser le contexte macro comme cadre explicatif pouvant avoir un impact sur la conception de l'enseignement des langues. Il en est un autre qui nous semble devoir être mis en avant et qui correspond à la culture didactique revendiquée comme inscription dans la formation proposée. Il semblerait que la «matrice disciplinaire » dans laquelle le groupe de la mention FLE s'inscrive, soit précisément celle de la didactique du FLE. Dans les exemples $4 a$. et $4 \mathrm{~b}$., il nous paraît probable que la différence d'appartenance didactique, c'est-à-dire le champ de la discipline dans lequel s'inscrit l'enseignant en formation, entre dans un système de représentation que nous signalons comme spécifique, à savoir un modèle idéalisé de la didactique du FLE qui a également fortement influencé la didactique des langues vivantes étrangères. Ce modèle didactique correspond aux solutions pédagogiques préconisées dans ce cursus, construit non pas en opposition au modèle de la didactique du FLM mais en référence aux méthodologies directe et/ou communicative qui la caractérisent. Nous en percevons la portée à travers les réponses concernant la place de la langue première dans l'apprentissage. En effet, afin de prolonger la réflexion sur le rôle de la langue première dans l'apprentissage, nous avons demandé à la suite du questionnaire initial de répondre de manière plus précise sur « la place à accorder à la langue première des élèves pendant la classe", afin de mieux évaluer dans quelle mesure celle-ci représentait un obstacle ou une richesse à exploiter.

12 Il apparaît que les enseignants de la mention FLE considèrent la L1 plus comme un obstacle à contourner qu'une richesse à exploiter.

\section{Exemple 4 : Corpus Mention FLE}

4.a Ens. 2: «Ce serait un frein, l'élève a sans doute l'occasion de pratiquer cette langue dans son cercle familial, il n'est pas nécessaire de l'intégrer dans son apprentissage. »

4.b Ens. 5: «Si je ne connais pas les langues que parlent mes élèves, je ne peux pas les aider, l'important est qu'ils avancent dans la langue. »

13 Ces exemples se caractérisent par leur inscription dans une culture didactique qui conçoit l'apprentissage des langues de manière séparée. Alors que les exemples précédemment cités reflètent plus une inquiétude quand au retard que cela implique lorsqu'on accorde une place à la L1 dans la classe, les affirmations qui suivent semblent marquer une certaine réticence vis-à-vis de son usage en classe tout en lui accordant un rôle actif dans l'apprentissage.

\section{Exemple 5 : Corpus DUDL}

5.a Ens.6 : «l'ignorer dans une certaine mesure pour permettre à l'élève de rentrer dans la langue à apprendre et lui permettre de construire des 
repères dans cette langue nouvelle, mais aussi l'intégrer car il me semble pas totalement négatif d'ignorer la langue maternelle car c'est celle dans laquelle l'enfant pense, ce qui peut faciliter l'apprentissage de la nouvelle langue. » 5.b Ens.8: "Tout dépend du contexte, l'ignorer pour éviter le recours systématique à la traduction dans un contexte où il y a une langue dominante et l'intégrer pour pouvoir faire des comparaisons entre les différentes grammaires dans les cas de forte hétérogénéité. » la L1 est considérée comme un point d'appui pour donner une impulsion à l'apprentissage en cours :

\section{Exemple 6 : Corpus DUDL}

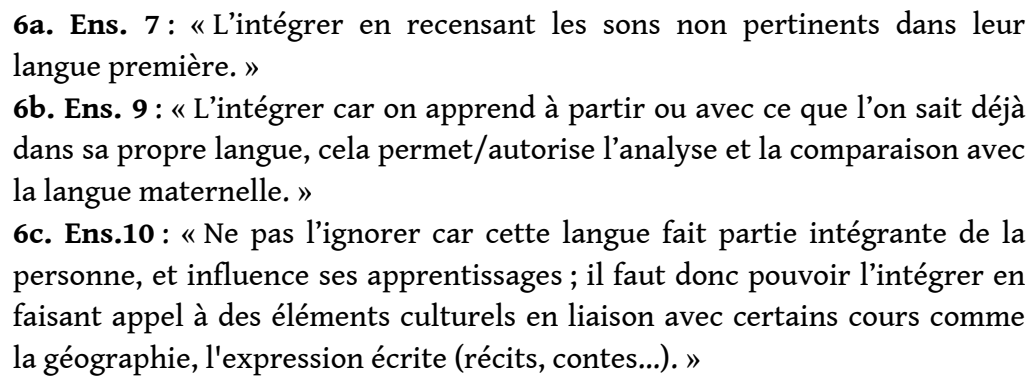
personne, et influence ses apprentissages ; il faut donc pouvoir l'intégrer en faisant appel à des éléments culturels en liaison avec certains cours comme la géographie, l'expression écrite (récits, contes...). »

Ces remarques semblent révéler, contrairement aux enseignants dont la culture éducative est ancrée dans une conception cloisonnée, que la L1 joue un rôle positif dans le domaine métacognitif, alors que les enseignants (exemples 5) dont la culture didactique se rattache à la didactique du français langue étrangère et des langues vivantes sont convaincus qu'il faut se détacher le plus rapidement possible de la L1 pour que le processus d'acquisition se déroule dans des conditions optimales. En conclusion, nous avançons l'idée que les enseignants du premier degré qui sont plus près d'une éducation au langage à l'école sont plus sensibilisés aux questions liées au développement psychoaffectif de l'enfant et semblent s'opposer aux objectifs que vise l'enseignement linguistique proposé dans le secondaire (Castellotti, 2001).

Alors que les étudiants des deux groupes semblent se rejoindre sur le fait que l'apprentissage en immersion est une situation idéale, il semble que pour être capable de réfléchir sur le rôle de la L1 dans l'appropriation, il faut en avoir bénéficié durant son propre apprentissage ou avoir été sensibilisé au rôle affectif de la L1 dans l'appropriation d'une L2. Or, à notre connaissance, les seules propositions qui nous semblent les plus efficaces pour valoriser le bi/plurilinguisme social dont il est question à l'heure actuelle, à travers la diffusion d'outils comme les Portfolios européens des langues, sont les démarches du type éveil aux langues qui réattribuent un rôle aux acquis linguistiques antérieurs.

\section{Entrer dans un processus de formation contextualisé}

17 A partir de ce recueil qui nous le savons n'a de valeur que dans le contexte dans lequel il a été recueilli, il est frappant de constater avec quelle conviction ces enseignants ou futurs enseignants de français langue étrangère ou seconde effectuent un certain nombre de choix théoriques et didactiques, peu en phase avec le scénario pédagogique et le contexte plurilingue dans lequel ils sont ou seront amenés à travailler. En effet, aujourd'hui 
l'évolution des sociétés contemporaines implique de plus en plus de travailler avec un public qui se caractérise par sa diversité linguistique mais on continue de penser dans notre système éducatif que la formation des enseignants en langues doit conduire à être un spécialiste d'une langue particulière, sans se préoccuper de la mise en place d'un véritable dispositif intégrant cette diversité dans la formation (Castellotti, 2001: 367, Alleman-Ghionda et al., 1999).

En effet, il n'existe pas dans les formations universitaires actuelles en didactique des langues, une réflexion d'ensemble sur la prise en compte de la diversité linguistique et culturelle comme proposition unifiée apportant des pistes didactiques adaptées aux classes où l'hétérogénéité linguistique est particulièrement représentée. Seules les universités du Mans, de Tours et certains Instituts Universitaires de Formation des maîtres (Grenoble, Bordeaux, Montpellier) ont mis en place un programme dont les contenus répondent à de tels objectifs. Ainsi, à travers l'expérimentation mise en place à l'université de Paris III, nous voulons rendre compte du fait que de nouvelles pistes de réflexion sont offertes quant à la prise en compte des langues premières des élèves qui ne sont pas valorisées socialement.

À la suite de notre étude empirique, il ressort que :

- Les enseignants (du premier et second degré) qui ne sont pas préparés à la diversité linguistique considèrent que la langue première peut être un frein dans l'apprentissage d'une langue seconde et/ou étrangère, contrairement aux enseignants qui y ont été sensibilisés.

- La diversité linguistique des élèves dans le contexte scolaire du français langue seconde et/ ou étrangère représente chez les enseignants plus une difficulté à contourner, qu'une richesse à exploiter.

- Il existe une relation entre la culture éducative à laquelle les enseignants appartiennent et la prise en compte de la diversité dans leurs pratiques.

- Les attitudes relatives à la diversité linguistique et culturelle relevées en début de formation sont susceptibles d'évoluer vers des propositions prenant en compte cette diversité en fin de formation.

Si du point de vue de la finalité éducative les deux modules de formation semblent équivalents, il semble que les enseignants inscrits en mention FLE qui ont reçu un enseignement orienté vers la dimension interculturelle de l'enseignement des langues accordent plus d'importance au regard que l'on porte sur l'autre, ce qui relève plus de l'adaptation du comportement et de la mise à distance de sa propre culture, alors que les enseignants du premier degré qui ont suivi une initiation à la diversité linguistique et culturelle sont plus attentifs au rôle positif que pourrait jouer la L1 dans l'apprentissage et à l'intérêt que représente un travail sur le fonctionnement des langues. En effet, l'étude des bilans de fin de parcours tend à montrer que des déplacements se sont opérés lorsqu'on les compare avec les représentations initiales et présentent du point de vue qualitatif de précieuses indications sur le cheminement parcouru. Si certains principes énoncés par la pédagogie interculturelle, tel qu'établir un dialogue entre les cultures sur la base d'un respect mutuel, s'avèrent en soi constructives, ces propositions s'avèrent pourtant insuffisantes sur le plan didactique pour travailler sur la diversité linguistique, comme ces extraits de bilans tendent à le montrer de manière contrastée en fonction des deux groupes :

\section{Exemple 7 : Extraits du bilan final}




\begin{abstract}
Corpus DUDL
7a. Ens.1 : "J'aimerais pouvoir utiliser d'autres mots et faire des activités permettant de passer d'une langue à d'autres, ce qui est très motivant. »

7b. Ens.3: «Je pratiquerais l'éducation à la tolérance par l'éveil aux langues. »

7c. Ens.4: "Je m'appuierais sur l'expérience linguistique plurielle qui permet d'associer à un signifiant plusieurs signifiés, et prendrait en compte progressivement des différences et ressemblances entre les langues rencontrées. »

7d. Ens.5: «Je commencerais par enrichir mes propres connaissances linguistiques et proposeraient des comparaisons entre les langues pour travailler sur le développement des capacités de réflexion et de représentation. Plus on rencontre de langues et de cultures, plus on peut accroître sa capacité d'ouverture par et envers les autres. »

7e. Ens.6: «Ce serait pour moi l'occasion de mieux me servir des connaissances des élèves en travaillant plus particulièrement sur les relations parents/école. »

Corpus Mention FLE

7f. Ens.1 : «Demander aux élèves s'ils ont voyagé, ce qu'ils ont trouvé de commun ou de différents par rapport à notre pays. »

7g. Ens.4: «Faire un travail préliminaire sur la situation sociolinguistique de notre pays, en proposant de constituer un historique des différentes langues et cultures qui ont composé et continue de composer la France. »

7h. Ens.5 : « S'ouvrir le plus possible aux autres langues en approfondissant toujours sa langue maternelle, tout en la préservant, essayer d'introduire des mots d'origine étrangère quand cela est possible. »

7i. Ens.6: «Se distancier de sa langue maternelle, en travaillant sur des textes littéraires provenant d'autres cultures. »
\end{abstract}

21 Comment un enseignant qui appartient à une culture éducative monolingue peut-il accroître son intérêt pour la diversité linguistique? Nous suggérons qu'une sensibilisation à la diversité linguistique et culturelle peut contribuer à asseoir les bases d'une réflexion commune entre apprentissage du français en classe linguistiquement hétérogène et prise en compte des langues premières à l'école pour créer un espace de contacts.

\title{
Vers une conception alterlinguistique de l'enseignement?
}

Nous avons vu que des connaissances en anthropologie culturelle font surgir un intérêt pour la découverte de l'autre, de sa langue et de sa culture mais nous pensons qu'il s'agit d'une première étape dans la prise en compte de la diversité linguistique et culturelle. En effet, une seconde étape serait celle de l'intégration des langues des élèves dans un curriculum d'éveil à la diversité linguistique. Comment les langues premières des élèves peuvent-elles intervenir dans la construction d'une didactique du contact au sein de la classe?

Cela nécessite un déplacement des frontières enseignant/enseigné dans laquelle l'enseignant intervient comme un passeur de langues et non comme un expert, en concentrant son activité sur la construction d'un savoir partagé et négocié entre enseignant et apprenants. Ces grands principes font référence aux propositions énoncées, 
d'une part, par Dabène (1992 : 20) qui souhaitait que les (futurs) professeurs d'école soient sensibilisés à une approche plurielle, "en suscitant, chez eux, à partir de leur propre acquis en langue maternelle comme en langue étrangère, une attitude de confrontation, d'ouverture et de découverte » et d'autre part, font écho aux propositions de Py qui préconisait la reconnaissance des savoirs que l'école n'a pas inculqués elle-même (1997 : 500) et qu'il serait pourtant utile de faire émerger pour éviter les manifestations d'insécurité linguistique constatées chez certains élèves. C'est à partir de ces considérations que nous souhaitons formuler quelques principes de cette conception qui constitueraient les bases d'une didactique du contact des langues (Véronique, $2005: 166$ ).

\section{Proposition pour une définition de la conception alterlinguistique de l'enseignement}

24 Nous entendons par cette conception, dépasser les principes de la pédagogie interculturelle en travaillant précisément à la mise en place d'une didactique du contact entre les langues. Ainsi, cette conception renvoie " aux moyens qui permettent de structurer les acquis sur le fonctionnement des langues à partir de l'expérience linguistique des apprenants, correspondant à l'ensemble des connaissances, savoirs et savoir-faire qui caractérisent l'appropriation des langues. » Elle s'articule autour des trois points suivants :

1) Prendre en compte l'apprenant dans sa globalité et sa spécificité en travaillant sur les biographies langagières :

Reconnaissance des apprenants comme interlocuteurs bi/plurilingues.

Reconnaissance de leur(s) identité(s) linguistique(s) et culturelle(s).

2) Faire face à l'altérité : repérage et vigilance

Vigilance lors de la perception de l'autre, éviter d'attribuer les caractéristiques associées aux catégorisations d'appartenance et aux stigmates assignés dans les représentations sociales (Goffman, 1975).

Repérage des stéréotypes et des représentations sociales et individuelles initiales liées aux langues, à leur apprentissage, ainsi qu'aux locuteurs de ces langues (Zarate, 1993).

3) Prendre conscience que :

L'apprentissage des langues ne relève pas uniquement d'une logique de rationalisation mais d'explicitation des différences culturelles et linguistiques.

L'apprentissage des langues ne s'inscrit pas dans un cadre normatif mais social.

Elle implique les compétences professionnelles suivantes :

- La prise en compte de la charge affective, sociale, identitaire et symbolique des langues au moment de l'enseignement/apprentissage.

- La maîtrise et la distanciation culturelle vis-à-vis de la langue d'enseignement et/ou d'apprentissage.

- La prise de conscience de l'enracinement culturel des langues.

- L'élucidation des rapports avec la culture d'appartenance ou de référence.

- La mise en œuvre éducative de la diversité linguistique.

Cette étude nécessite encore une recherche plus approfondie en ce qui concerne le véritable degré d'impact du système éducatif français d'apprentissage des langues pour confirmer notre hypothèse. Bien que nos résultats semblent confirmer qu'il existe un désir de mieux connaître les langues des élèves et de sortir d'une vision dichotomique des langues, il est difficile de substituer une culture didactique à une autre, dans une école où l'on propose de remplacer, dans le cas de l'apprentissage du français langue seconde, un 
monolinguisme par un autre et dans le cas de l'apprentissage d'une langue étrangère, de « cumuler deux monolinguismes » (voir Ducancel \& Simon (dir.), 2004).

\section{En guise de conclusion}

La conclusion la plus importante de notre étude réside dans le fait que les enseignants et futurs enseignants sont davantage conscients des points sur lesquels il faut appuyer pour réattribuer un rôle positif à la L1, en découvrant notamment comment mettre en œuvre la diversité linguistique et culturelle dans leur classe, ce qui n'est peut être pas suffisant lorsqu'ils sont sensibilisés à une approche de la pédagogie interculturelle.

En reconnaissant l'importance du travail cognitif que l'apprenant met en œuvre lorsqu'il apprend une langue, nous pensons qu'un rapprochement entre la didactique des langues premières et des langues secondes et/ou étrangère est possible. Il nous semble que les approches plurielles telles que définies dans les propositions d'Éveil aux langues pourraient contribuer à la constitution d'une culture didactique commune dans la formation des enseignants de langues. En effet, nous avons montré que l'identité de l'enseignant ou futur enseignant évolue, au-delà de la culture éducative à laquelle il appartient lorsqu'il est confronté à un nouveau modèle de culture didactique, ancré dans un contexte socio-éducatif plus en phase avec la réalité hétérogène qu'il côtoie ou côtoiera.

\section{BIBLIOGRAPHIE}

Abdallah-Pretceille, M. (2004). « L'éducation interculturelle ». Que sais-je ?, n 3487 (1 ${ }^{\text {ère }}$ ed. 1999). Paris : PUF.

Alleman-Ghionda, C., Perregaux, C. \& De Goumoëns, C. (1999). Curriculum pour une formation des enseignants. Berne : Programme national de recherche 33 \& Aarau, Centre suisse de la recherche en éducation.

Candelier, M. (dir.) (2003). L'éveil aux langues à l'école primaire. Evlang: bilan d'une innovation européenne. Bruxelles : De Boeck.

Castellotti, V. (2001). La langue maternelle en classe de langue étrangère, Paris : CLE International.

Castellotti, V. \& Moore D. (2002). Représentations sociales des langues et enseignements. Politique linguistique, Strasbourg : Conseil de l'Europe.

Dabène, L. (1992). « Le développement de la conscience métalinguistique : un objectif commun pour l'enseignement de la langue maternelle et des langues étrangères ». Repères, $n^{\circ}$ 6. pp. 13-21. INRP.

Develay, M. (1992). De l'apprentissage à l'enseignement. Paris : ESF.

Ducancel, G. \& Simon, D.-L. (dir.) (2004), « Français et langues étrangères et régionales à l'école? Quelles interactions? ». Repères, $\mathrm{n}^{\circ} 29$. INRP. 
Hawkins, E. (1992). « La réflexion sur le langage comme « matière-pont » dans le programme scolaire ». Repères, $\mathrm{n}^{\circ}$ 6. pp. 41-56. INRP.

Moore, D. (dir.) (2003). Les représentations des langues et de leur apprentissage, référence, modèles, données et méthodes. Paris : Didier-Collection Credif/Essais.

Perrenoud, P. (2003). Développer la pratique réflexive dans le métier d'enseignant. Paris : ESF (1 ${ }^{\mathrm{èr}}$ ed. 2001).

Porquier, R., \& Py, B. (2004). Apprentissage d'une langue étrangère : contexte et discours. Paris : DidierCollection Credif/Essais.

Puren, L. (2004). L'école face à l'enfant alloglotte entre 1880 et 1985. Thèse DNR, Université Paris III.

Py, B. (1997). « Pour une perspective bilingue sur l'enseignement et l'apprentissage des langues ». Études de linguistique appliquée, $\mathrm{n}^{\circ}$ 108. Paris : Didier Erudition.

Rémy-Thomas, F. (à paraître). « Toutes les langues à l'école ou comment dépasser les frontières institutionnelles pour créer un espace de contacts ». Actes de la journée scientifique internationale " Contacts des espaces, contacts des langues - Entre frontières naturelles et frontières artificielles ", Diltec (EA 2288), Université de la Sorbonne Nouvelle - Paris III. Synergie.

Sabatier, C. (2004). Rôle de l'école dans le développement et la construction du plurilinguisme chez des enfants issus de la migration maghrébine en France. Thèse DNR, Université Stendhal - Grenoble 3.

Véronique, D. (2005). «L'apprentissage de la langue et les appartenances langagières multiples : aspects d'une politique linguistique éducative ». Le français hier et aujourd'hui, Politiques de la langue et apprentissages scolaires. Aix-en-Provence : PUP.

\section{ANNEXES}

\section{Questionnaire initial}

Question 1 : Quelle est selon vous la meilleure façon d'apprendre une langue?

Question 2 : A quoi sert l'apprentissage d'une/des langues?

Question 3 : Selon vous, quelle place doit-on accorder à la langue première des élèves pendant la classe?

- l'ignorer, Pourquoi?

- l'intégrer, Comment?

\section{Question 1 : Quelle est selon vous la meilleure façon d'apprendre une langue ?}

\section{Corpus Mention FLE (enseignants ou futurs enseignants du second degré)}

Ens. 1 : Communiquer avec ses locuteurs [de la L2] et séjourner dans un lieu où elle est pratiquée rendent l'apprentissage plus vivant et naturel. Pas d'expérience de l'enseignement du français et/ou d'une LVE au collège/lycée.

Ens. 2 : Je pense que pour apprendre correctement une langue la meilleure façon est de partir dans le pays où l'on parle cette langue et de vivre avec les gens de ce pays.

Expérience de l'enseignement du français dans une association (2 ans). 
Ens. 3 : La meilleure façon d'apprendre une langue c'est de se rendre dans le pays où cette langue est pratiquée. Expérience de l'enseignement du français dans un lycée français (5 ans).

Ens. 4 : La meilleure façon est pour moi de s'installer dans le pays où elle est parlée et de s'immerger totalement, en écoutant la radio et la T.V dans cette langue, de lire les journaux et des livres. Expérience de l'enseignement du français au collège ( 2 ans en CLA).

Ens. 5 : Habiter le pays où l'on parle cette langue et s'immerger dans la culture locale. Pas d'expérience de l'enseignement du français au collège/lycée.

Ens. 6 : Selon moi, les meilleures façons d'apprendre une langue sont d'aller dans le pays pour quelques temps ou que la langue soit enseignée par un locuteur natif. Expérience de l'enseignement du français en lycée professionnel (14 ans).

Ens. 7 : Il faut être avec des personnes qui parlent cette langue et ne parlent pas la mienne pour me forcer à parler. Expérience de l'enseignement du français au collège/ lycée (10 ans).

Ens. 8 : L'immersion dans le pays est selon moi la meilleure façon de connaître une langue. Expérience de l'enseignement du français au collège/lycée (8ans).

Ens. 9 : Partir pour une durée de 6 mois ou 1 an minimum à l'étranger afin de maîtriser la langue en question en immersion totale, c'est-à-dire en communiquant avec les gens de ce pays du matin au soir, en évitant au maximum de parler sa langue maternelle avec des compatriotes rencontrés dans le pays. Pas d'expérience de l'enseignement du français et/ ou LVE au collège/lycée.

Ens. 10 : La meilleure façon est de s'immerger totalement dans le pays en ne pouvant parler avec personne notre langue maternelle. Expérience de l'enseignement du français au collège/lycée (5 ans).

\section{Corpus DUDL (enseignants ou futurs enseignants du premier degré)}

Ens. 1 : de façon ludique, en immersion, à l'aide de supports divers, par la répétition. Expérience de l'enseignement des langues aux enfants en institution scolaire (4 ans).

Ens. 2 : travailler les mots avec son corps, en parlant et écoutant, s'ouvrir à tous les aspects de la langue étrangère. Expérience de l'enseignement des langues aux enfants en institution scolaire (22 ans).

Ens. 3 : être immergé pour parler, avoir un bon livre de grammaire pour écrire. Expérience de l'enseignement des langues aux enfants en institution scolaire (5ans).

Ens. 4 :

- À l'oral : par le jeu (jeux de rôles, de gestes, de sons, marionnettes, chants) et la communication. En groupe, dans des situations d'échange et dans des situations de la vie où il $\mathrm{y}$ a répétitions d'actions ritualisées ou non.

- À l'écrit: par le dessin, puis chercher les mots dans des imagiers ou des dictionnaires. Dans la correspondance oral/écrit. Expérience de l'enseignement des langues aux enfants en institution scolaire (15 ans dont 4 en Clin) 
Ens. 5 : être immergé, en avoir l'utilité et en éprouver du plaisir. Si ce n'est pas le cas, lors de situation d'échec, être accompagné pour surmonter ses difficultés. Pas d'expérience de l'enseignement des langues aux enfants.

Ens. 6 : en créant de situations de communication authentiques dans lesquelles un besoin nouveau, en termes de vocabulaire, apparait. Expérience de l'enseignement des langues aux enfants en institution scolaire (25 ans).

Ens. 7 : Vivre dans le pays où l'on parle cette langue. Expérience de l'enseignement des langues aux enfants en institution scolaire (12 ans).

Ens. 8 : par la pratique, l'écoute et la lecture. Expérience de l'enseignement des langues aux enfants en institution scolaire (6 ans).

Ens. 9 : immersion par degrés sécurisés à chaque palier. Pas d'expérience de l'enseignement des langues aux enfants.

Ens. 10 : par une activité extra-scolaire (jeu, théâtre, chant, sport, dessin). Expérience de l'enseignement des langues aux enfants en institution scolaire ( 6 ans puis reconversion professionnelle).

\section{Bilan de fin de parcours : Quelles propositions feriez-vous pour mieux prendre en compte la diversité linguistique et culturelle dans votre pratique?}

\section{Corpus Mention FLE :}

Ens. 1 : Demander aux élèves s'ils ont voyagé, ce qu'ils ont trouvé de commun ou de différents par rapport à notre pays.

Ens. 2 : Pour s'approprier une langue, il faut comprendre tout ce qu'elle véhicule et c'est en proposant de la mettre en situation que l'on fera accepter la charge affective et sociale qu'elle dégage.

Ens. 3 : Chaque individu, en parlant sa langue, contribue à la faire vivre et ainsi la faire partager aux autres, ce qui suppose de leur accorder une place dans la classe.

Ens. 4 : Faire un travail préliminaire sur la situation sociolinguistique de notre pays, en proposant de constituer un historique des différentes langues et cultures qui ont composé et continue de composer la France.

Ens. 5 : S'ouvrir le plus possible aux autres langues en approfondissant toujours sa langue maternelle, tout en la préservant, essayer d'introduire des mots d'origine étrangère quand cela est possible.

Ens. 6 : Se distancier de sa langue maternelle, en travaillant sur des textes littéraires provenant d'autres cultures.

Ens. 7 : Je mettrais en place des ateliers décloisonnés avec des enseignants de langues.

Ens. 8 : Je favoriserais la compréhension mutuelle par les échanges interculturels, en favorisant la tolérance dans le groupe, en laissant une grande part au travail d'expression artistique.

Ens. 9 : Travailler sur les représentations pour se décentrer par rapport à sa propre culture, son système éducatif... 
Ens. 10 : Dépasser son ignorance face aux langues des élèves en tirant partie de cette richesse.

\section{Corpus DUDL}

Ens. 1 : J'aimerais pouvoir utiliser d'autres mots et faire des activités permettant de passer d'une langue à d'autres, ce qui est très motivant.

Ens. 2 : Je travaillerais à la mise en place d'échanges multiples entre les élèves et l'enseignant.

Ens. 3 : Je pratiquerais l'éducation à la tolérance par l'éveil aux langues.

Ens. 4 : Je m'appuierais sur l'expérience linguistique plurielle qui permet d'associer à un signifiant plusieurs signifiés, et prendrait en compte progressivement des différences et ressemblances entre les langues rencontrées.

Ens. 5 : Je commencerais par enrichir mes propres connaissances linguistiques et proposeraient des comparaisons entre les langues pour travailler sur le développement des capacités de réflexion et de représentation. Plus on rencontre de langues et de cultures, plus on peut accroître sa capacité d'ouverture par et envers les autres.

Ens. 6 : Ce serait pour moi l'occasion de mieux me servir des connaissances des élèves en travaillant plus particulièrement sur les relations parents/école.

Ens. 7 : Par une sensibilisation à la différence de l'autre, je pense qu'il est possible de travailler sur les difficultés des apprenants. « Je me souviens d'un apprenant serbe, Milan, qui disait sans arrêt que le français était trop difficile à apprendre, qu'il n'y arriverait jamais. Un apprenant sri lankais lui a répondu que si l'alphabet français comportait 26 lettres, l'alphabet de sa langue maternelle, le tamoul, en comportait 320. Milan s'est remit vaillamment à l'ouvrage, sans plus jamais se décourager ».

Ens. 8 : Par la mise en place de jeux dans la classe, d'enquêtes et d'ateliers autour d'activités comme celles de l'éveil aux langues.

Ens. 9 : En proposant d'établir de nombreux ponts linguistiques et culturels.

Ens. 10 : On peut soutenir l'intérêt et la curiosité des apprenants grâce à la confrontation des expériences linguistiques et des univers culturels en travaillant en groupes et ensuite formaliser les confrontations entre ces langues à l'oral ou à l'écrit.

\section{NOTES}

1. Corpus recueilli durant l'année universitaire 2003-2004. Cette mention a été remplacée depuis l'entrée en vigueur du LMD (en vue d'une harmonisation des diplômes au niveau européen) par un parcours de didactique du français au niveau L à l'université Paris III.

2. Corpus recueilli durant l'année universitaire 2004-2005. Ce diplôme est désormais intégré pour partie dans le parcours du M (master professionnel, ex DESS).

3. Pour une définition plus précise de cette notion, voir Cadet dans cette publication et Beacco et al (Eds.), (2005). Comprendre les cultures éducatives. Paris : Presses Universitaires de France.

4. Nous faisons référence à la question de l'idéal monolingue qui caractérise l'histoire de l'unification linguistique de la France et qui s'est traduite dans les faits par la position dominante du français vis-à-vis des "autres" langues présentes à l'école (voir Puren, 2004). 
5. Voir sur ce sujet Abdallah-Pretceille (1999) et pour situer l'histoire de cette pédagogie dans l'évolution de la prise en compte de l'altérité linguistique et culturelle à l'école dans Sabatier 2004.

6. Voici la définition qu'ils en donnent (2004: 59) : "Le niveau macro comporte les déterminations sociales au sens le plus large, telles que : les politiques éducatives, les politiques linguistiques des pays concernés, les systèmes éducatifs eux-mêmes...le niveau micro correspond à des moments ou à des séquences de dimensions variables mais comportant une unité de temps, de lieu et d'interaction." Voir aussi l'article de Varshney dans cette publication.

7. Voir en annexe 1, le questionnaire distribué destiné à orienter la réflexion sur les notions et concepts clés du cours, dans le but de «Développer la pratique réflexive dans le métier d'enseignant ». Cette proposition est une reprise du titre d'un ouvrage de Perrenoud (2001).

8. L'expression "matrice disciplinaire" est initialement définie par Kuhn (1969) à propos des savoirs scientifiques et a été reprise par Develay (1992) qui prend appui sur cette conception pour la transférer à l'enseignement : "les matrices disciplinaires" prennent forme à travers des connaissances déclaratives, des connaissances procédurales, des tâches, des objets.

9. Coste \& Moore (1994 : 412) parlent de "rituels d'apprentissage" pour désigner l'entraînement académique des adultes à propos de leur parcours d'apprenant.

10. Pour un rappel des travaux sur ce sujet, voir Varshney dans cette publication.

\section{RÉSUMÉS}

Contrairement à ce que la didactique des langues a longtemps contribué à ancrer dans les esprits, la langue première ne contrarie pas l'apprentissage d'une langue seconde (voir Castellotti, 2001). De fait, sa présence implique que l'on réfléchisse à la mise en œuvre de nouvelles dynamiques d'enseignement/apprentissage. Dans cet article, nous nous proposons d'étudier comment des enseignants ou futurs enseignants font évoluer leur conception idéalisée de l'apprentissage des langues face à la diversité linguistique et culturelle. Il s'agira d'analyser comment la réalité des classes composées d'élèves d'origines linguistiques diverses conduit à un repositionnement de l'enseignement qui consisterait à : placer l'élève au centre de l'apprentissage en lui reconnaissant le statut de personne bi/plurilingue, lui permettre de devenir co-acteur du savoir qu'il s'approprie, en cherchant à soutenir le travail de comparaison entre les langues (projet Evlang, Candelier, 2003) et l'aider à entrecroiser les langues de son répertoire, plutôt qu'à les cloisonner (Castellotti, $2001: 80$ ).

Despite popular and long-held beliefs, the first language does not stand in the way of second language acquisition (Castelotti 2001). In fact, the use of the L1 in the language classroom may give rise to a new dynamics of teaching and learning. In this article, we will examine how teachers and teacher trainees faced with linguistic and cultural diversity are forced to develop new ideas about language teaching. Classrooms composed of students from diverse linguistic backgrounds create require a new agenda for language teachers: placing the students at the center of the learning process; recognizing their plurilingualism; enabling them to become an active participants in building up new language knowledge, by comparing their different languages (Candelier 2003); and helping them combine the languages in their repertoires, rather than partitioning them (Castellotti 2001: 80). 
INDEX

Mots-clés : diversité linguistique et culturelle, altérité linguistique, bi-plurilinguisme, formation Keywords : alterlinguistics, teaching in a context of linguistic diversity

\section{AUTEUR}

\section{FLORENCE RÉMY-THOMAS}

Diltec, université Sorbonne nouvelle - Paris III, France 